\title{
Localization transition of instantaneous normal modes and liquid diffusion
}

\author{
Vasile Iulian Clapa, Tsampikos Kottos, and Francis W. Starr \\ Department of Physics, Wesleyan University, Middletown, Connecticut 06459, USA
}

(Received 18 January 2012; accepted 22 March 2012; published online 12 April 2012)

\begin{abstract}
We analyze the structure of instantaneous normal modes (INM) associated with the Hessian matrix of a liquid. Utilizing a scaling theory developed in the framework of Anderson localization studies, we unambiguously identify the crossover point in the INM spectrum between extended and localized modes. We establish a relation between the unstable, delocalized INMs and the liquid diffusion coefficient that appears valid over a large temperature range, covering both Arrhenius and non-Arrhenius regimes of temperature dependence. These results suggest a possible route to theoretically relate dynamics to thermodynamical properties of the liquid via the tomography of the INMs. @ 2012 American Institute of Physics. [http://dx.doi.org/10.1063/1.3701564]
\end{abstract}

\section{INTRODUCTION}

Computer simulations have played an increasingly important role in explaining the molecular mechanisms controlling the dynamics of supercooled liquids approaching their glass transition temperature $T_{g}{ }^{1-3}$ Studies on the potential energy surface (PES) directly connect the slowing down of the dynamics and the changes of topological features of PES. ${ }^{3-6}$ For example, the local curvature of the PES influences the directions in configuration space that the liquid can readily explore. In analogy with solids, where the curvature of the PES in the vicinity of a minimum defines the normal modes, we can evaluate the instantaneous normal modes (INM) of a liquid in the vicinity of an arbitrary liquid configuration., ${ }^{7,8}$ The INMs are obtained from the eigenvalues of the Hessian matrix, whose elements are the second derivatives of the potential energy with respect to the atomic coordinates. In the case of liquids, there is a non-negligible number of negative eigenvalues, which are associated with downward curvatures in the PES. Such negative curvatures represent the unstable directions in configuration space, and in analogy with the normal mode picture, have imaginary mode frequency. The number of unstable directions decreases on cooling, and it was conjectured $^{9,10}$ that there is a connection between diffusion of liquids and the number of unstable modes.

Given that even deeply quenched states with no mobility exhibit a finite fraction of unstable eigenmodes, it is clear that not all negative eigenvalues are associated with the long-time dynamics. ${ }^{11-13}$ Consequently, a variety of filtering techniques of the imaginary modes to find those related to diffusion have been proposed. ${ }^{12-20}$ In particular, we focus on a proposal by Bembenek and Laird ${ }^{12,13}$ to filter unstable modes by examining the fraction of the system participating in the mode; they argued that only those unstable modes that are delocalized relate to diffusion. Delocalized modes involve an extensive fraction of the system, while localized modes involve a finite, intensive fraction of the system. Accordingly, one expects that (in the thermodynamic limit) a localized mode can only contribute to the rearrangement of a vanishingly small fraction of the fluid, and hence will not contribute to the overall diffusion. In contrast, since delocalized modes extend over the entire system, they can contribute to large scale rearrangement that leads to diffusion. This point has been already recognized in the frame of mesoscopic transport phenomena, where researchers have connected the structure of eigenmodes with the transport properties (conductance) of random media. $^{21}$

While this approach has shown promise for fluids, attempts to unambiguously separate delocalized modes $^{12,13,22,23}$ have not succeeded to identify a unique crossover between localized and delocalized modes.

A similar analysis has matured in the studies of mesoscopic transport in random media, where methods based on random matrix theories (RMT) (Refs. 24 and 25) have been utilized to develop scaling techniques that allow for a detailed description of the structure and localization properties of the normal modes associated with these media. ${ }^{26}$ These theories assume that the statistical properties of complex random media can be effectively described by an ensemble of random matrices which do not keep the details but only the symmetry of the original problem. The validity of RMT has been tested successfully during the last 30 years, via both numerical and direct experimental studies. ${ }^{21,27-29}$

Since the elements of the Hessian are deterministic functions of random positions, these elements can be treated as random variables. Thus, following the RMT philosophy, the Hessian matrix can be classified as an example of a recently introduced RMT ensemble, termed Euclidean random matrices (ERMs). ${ }^{30}$ Based on this observation, we use RMT methodologies developed in the frame of mesoscopic transport, as a way to aid in separating the localized and delocalized modes in our model liquid system. Specifically, we show that we can identify the transition point between the localized and delocalized modes of the Hessian by a finite size scaling approach; the transition can be located with surprising small system sizes. We use this test to filter the modes that are both delocalized and follow unstable directions in configuration space. We find that the fraction $f_{u}^{\text {DL }}$ of these modes can be related to the liquid diffusion coefficient $D$ over a large temperature range, covering both Arrhenius and non-Arrhenius regimes. 
The structure of the paper is as follows: in Sec. II, we discuss the model and simulation methods; in Sec. III, we make use of the methods developed in the framework of mesoscopic transport in disordered media to distinguish mode types of the Hessian; in Sec. IV, we propose a connection between the delocalized fraction of INMs and the diffusion coefficient. Our results are summarized in Sec. V.

\section{MODEL AND SIMULATION METHODS}

Our results are based on equilibrium molecular dynamics simulations of the binary Lennard-Jones mixture, popularized by the work of Kob and Andersen (KA). ${ }^{31,32}$ This mixture was inspired by a model introduced by Weber and Stillinger ${ }^{33}$ for the study of the glass transition of a metallic glass former, $\mathrm{Ni}_{80} \mathrm{P}_{20}$. This has become the standard molecular model to study glass formation for simple fluids, and its thermodynamic and dynamic properties have been extensively catalogued. ${ }^{31,32,34-36}$ Accordingly, it is an ideal system to examine carefully the localization properties of the INMs.

The KA model consists of an 80:20 mixture of $\mathrm{A}$ and B particles that have identical mass $m$ and interact via a Lennard-Jones potential

$$
V_{\alpha \beta}(r)=4 \epsilon_{\alpha \beta}\left[\left(\frac{\sigma_{\alpha \beta}}{r}\right)^{12}-\left(\frac{\sigma_{\alpha \beta}}{r}\right)^{6}\right],
$$

where $\alpha, \beta \in\{A, B\}$; here $\epsilon_{A A}=1.0, \sigma_{A A}=1.0, \epsilon_{A B}$ $=1.5, \sigma_{A B}=0.8, \epsilon_{B B}=0.5$, and $\sigma_{B B}=0.88$. The potential is truncated and shifted at a cutoff distance of $2.5 \sigma_{\alpha \beta}$. We report our results in reduced units where length is in units of $\sigma_{A A}$, temperature in units of $\epsilon_{A A} / k_{\mathrm{B}}$ (where $k_{\mathrm{B}}$ is Boltzmann's constant), and time in units of $\left(m \sigma_{A A}^{2} / \epsilon_{A A}\right)^{1 / 2}$. All simulations are at a fixed density of $\rho=1.204$ with periodic boundary conditions, which corresponds to the canonical isocore studied in the literature. ${ }^{31,32}$ We consider a broad temperature range $0.44 \leq T \leq 2.5$, covering both normal and "supercooled" ranges; temperature is controlled using the deterministic Nosé-Hoover thermostat. ${ }^{37}$ We confirmed that the diffusion coefficient $D$ and structural properties match those reported in Refs. 31 and 32 (although note that the unit of time we use differs slightly from that of Refs. 31 and 32). In order to study the finite size effects on the INM, simulations have been performed for system sizes $N=200,400,800,1000$, and 1600 particles.

\section{LOCALIZATION TRANSITION OF THE INM}

\section{A. Properties of the Hessian matrix}

The investigation of the statistical properties of the normal modes of the Hessian matrices $H$ as a tool to understand liquid dynamics has a long history. ${ }^{7-20,22,23,35,38-42}$ These matrices characterize the curvatures of the total potential energy surface. Formally, the Hessian $H$ is a $3 N \times 3 N$ matrix defined from the potential energy $V(\vec{r})$,

$$
H_{i \alpha, j \beta}=\frac{\partial^{2} V}{\partial r_{i \alpha} \partial r_{j \beta}},
$$

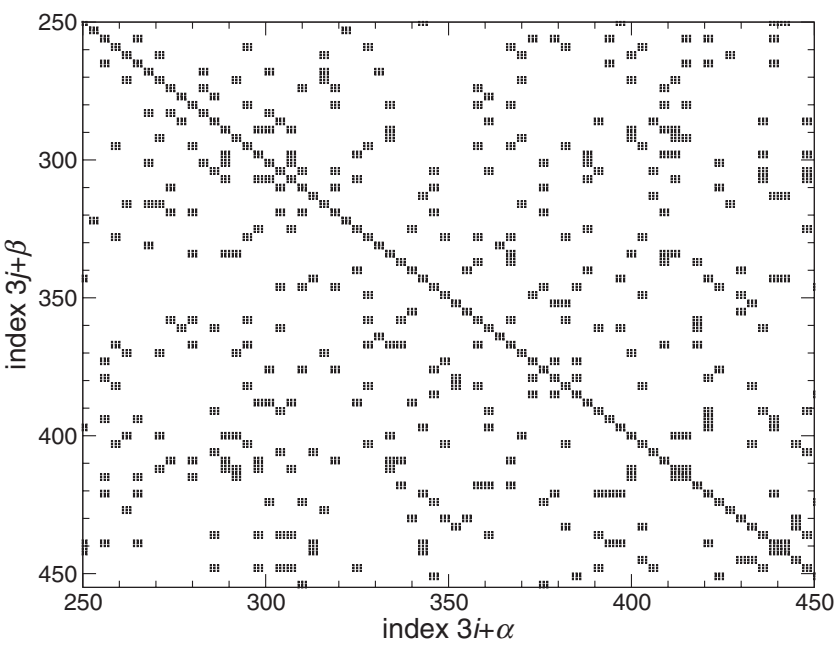

FIG. 1. Illustration of the structure of the Hessian. The non-zero elements of the Hessian are marked by dots, while the zero elements are left blank. Note that we show only a small range of a $3000 \times 3000$ Hessian to facilitate visualisation. The indices $i$ and $j$ refer to particle number, while $\alpha$ and $\beta$ refer to Euclidean directions, taking values 1, 2, or 3; see Eq. (2).

where $i$ and $j$ are particle indices and $\alpha$ and $\beta$ are indices for spatial direction; the components of $H$ are the second-order coefficients of an expansion of the system's potential energy about a given configuration.

The Hessian matrix looks like a random matrix (Fig. 1), with randomness originating from the disorder in the position of the particles. In this respect, the Hessian matrices of liquid systems can be considered as a generalized version of Euclidean random matrices. ${ }^{23}$ This is a category of RMT models that falls under the same universality class with sparse random matrices describing amorphous disordered media. ${ }^{43,44}$

The matrix elements of $H$ satisfy a number of constrains due to Newtonian conservation laws. These constrains introduce correlations between the matrix elements; additionally, the sparsity of $H$ increases weakly on cooling. While such correlations between matrix elements are not present in traditional RMT models used in mesoscopic systems, the effect of sparsity on the transport properties of random media has been investigated in the past literature. ${ }^{45-47}$ These studies indicated that the structure of the normal modes of such disordered media undergo a transition from extended modes (extensive in system size) to completely localized modes (intensive in system size); at this transition, a complete halt of transport can be observed. The phenomenon, termed the Anderson metalto-insulator phase transition (MIT), has its origin in destructive wave interference and was first predicted 60 years ago by Anderson. ${ }^{48}$

The corresponding normal mode problem associated with the Hessian matrix (2) reads

$$
H \mathbf{e}_{\mathbf{n}}=\lambda_{n} \mathbf{e}_{\mathbf{n}} ; \quad \lambda_{n}=m \omega_{n}^{2},
$$

where $\omega_{n}$ are the INM frequencies, $m$ is the particle mass, and $\mathbf{e}_{\mathbf{n}}$ are the corresponding eigenvectors. For solids, the expansion in Eq. (2) is done around a stable point, so that the $3 N$ eigenfrequencies $\omega_{n}$ represent the vibrational normal mode frequencies in the harmonic limit. The INMs for the liquid state are obtained in the same way, except that $H$ is 

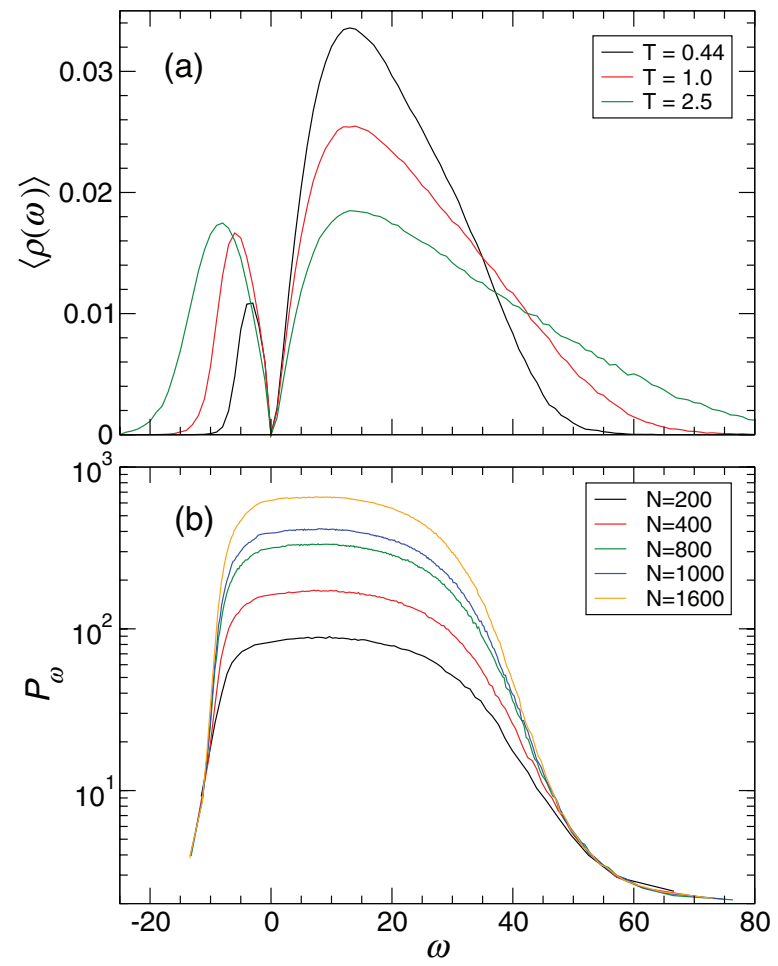

FIG. 2. (a) The INM density of states for representative $T=0.44,1.0,2.5$. (b) Typical participation number $P_{\omega}$ for $T=1.0$ and various system sizes.

evaluated from an arbitrary equilibrium liquid configuration. Since the typical liquid configuration is not a local minimum of the potential energy, not all the eigenvalues $\lambda_{n}$ of $H$ are positive. One then can identify the negative part of the spectrum with the unstable directions in configuration space. Using the analogy with a system of coupled oscillators, these negative eigenvalue modes are associated with the existence of negative coupling constants, which can be interpreted as the square of an imaginary frequency. Hence the INMs are classified as stable ( $\omega$ real) and unstable ( $\omega$ imaginary). Following standard practice, we represent the imaginary frequency modes as negative frequencies, i.e., $-\omega$ represents an imaginary frequency mode $i \omega$, to facilitate data presentation.

The natural starting point is to consider the INM density of states (DOS) $\langle\rho(\omega)\rangle$. To evaluate $\langle\rho(\omega)\rangle$ (and all subsequent INM quantities), at each $T$ we sample $H$ from 100 evenly spaced equilibrium configurations, each separated by approximately the structural relaxation time at the given temperature $T$. Averages are evaluated from these independent samples. Note that the eigenvalues include three zero frequency modes that correspond to free translation of the system; we remove these three trivial modes from all analysis. In Fig. 2, we show $\langle\rho(\omega)\rangle$ for representative temperatures, $T=0.44,1,2.5$, covering the range from supercooled to supercritical liquid states of our system. Cooling toward $T_{g}$, the decrease in mobility is accompanied by a decrease in the fraction of unstable modes $f_{u}$, apparent from the decrease in the negative lobe of $\langle\rho(\omega)\rangle$ (Fig. 2). This has long been considered an indicator that the unstable modes are related to the diffusivity of the system.
While there is a coarse correspondence between $f_{\mathrm{u}}$ and $D$, it is known that not all unstable modes relate to paths promoting diffusion. ${ }^{12-14,17}$ This is apparent from the fact that systems with virtually no diffusion - such as deeply quenched glasses or crystalline states - still have a non-negligible fraction of unstable modes. The explanation of this fact is that some of these unstable directions are not diffusive, and represent local anharmonicities of vibrational motion.

To help separate the modes that are perhaps more directly related to diffusion, we will make use of scaling methods developed in the framework of Anderson localization theory for the investigation of MIT.

\section{B. Participation number and localization of INMs}

To separate the extended from localized INMs, we consider a direct investigation of the spatial extent of the eigenvectors. Drawing on the analogies between the RMT description of the Anderson problem, we expect that the INMs of our system can be divided in two broad categories: (i) localized, meaning that the number of particles participating in a mode is intensive, or (ii) delocalized, meaning that the number of particles participating in a mode is extensive. To quantify the degree of localization, we use the participation number $(\mathrm{PN}),{ }^{26,49}$ which for particulate systems is expressed by ${ }^{50}$

$$
P_{\omega}=\left\langle\left(\sum_{i}\left(\mathbf{e}_{i}^{\omega} \cdot \mathbf{e}_{i}^{\omega}\right)^{2}\right)^{-1}\right\rangle,
$$

where $\mathbf{e}_{i}^{\omega}$ is the contribution of particle $i$ to the normalized eigenvector corresponding to eigenfrequency $\omega$.

Figure 2(b) shows $P_{\omega}$ for all $N$ studied at $T=1.0$. One can immediately recognize that $P_{\omega}$ of INMs near the middle of the DOS scales proportionally to the size of the system $\mathcal{O}(N)$ (delocalized modes); in contrast, for the modes at the tails, $P_{\omega}$ converges to a finite value that is proportional to a characteristic length-scale of the localization of the eigenmode. Indeed, Bembenek and Laird ${ }^{12,13}$ found preliminary evidence that the modes from the tails of the INM DOS are localized; they proposed that vitrification occurs when all unstable modes are localized.

To pinpoint the transition frequency from localized to delocalized behavior, we consider the finite-size scaling approach introduced by Ref. 49 for the study of the MIT phase transition in three-dimensional amorphous media. These authors showed that a MIT crossover can be identified by considering the participation number scaled by the linear system size $L \sim N^{1 / 3}$, i.e., $\widetilde{P}_{\omega} \equiv P_{\omega} / L$. According to Ref. 49 , at the transition point (mobility edge) the scaled PN $\widetilde{P}_{\omega}$ is $L$-invariant.

Figure 3 shows that indeed there are fixed points of $\widetilde{P}_{\omega}$ near the tails of the spectrum. These fixed points identify the location of the crossover frequency $\omega_{c}$ between localized and delocalized modes. Hence, we have found a novel way to unambiguously separate localized and delocalized modes in the liquid state using rather small system sizes $\left(\mathcal{O}\left(10^{3}\right)\right)$. For comparison, we visualize the contribution of each particle to $P_{\omega}$ for an arbitrarily chosen localized and delocalized mode for 

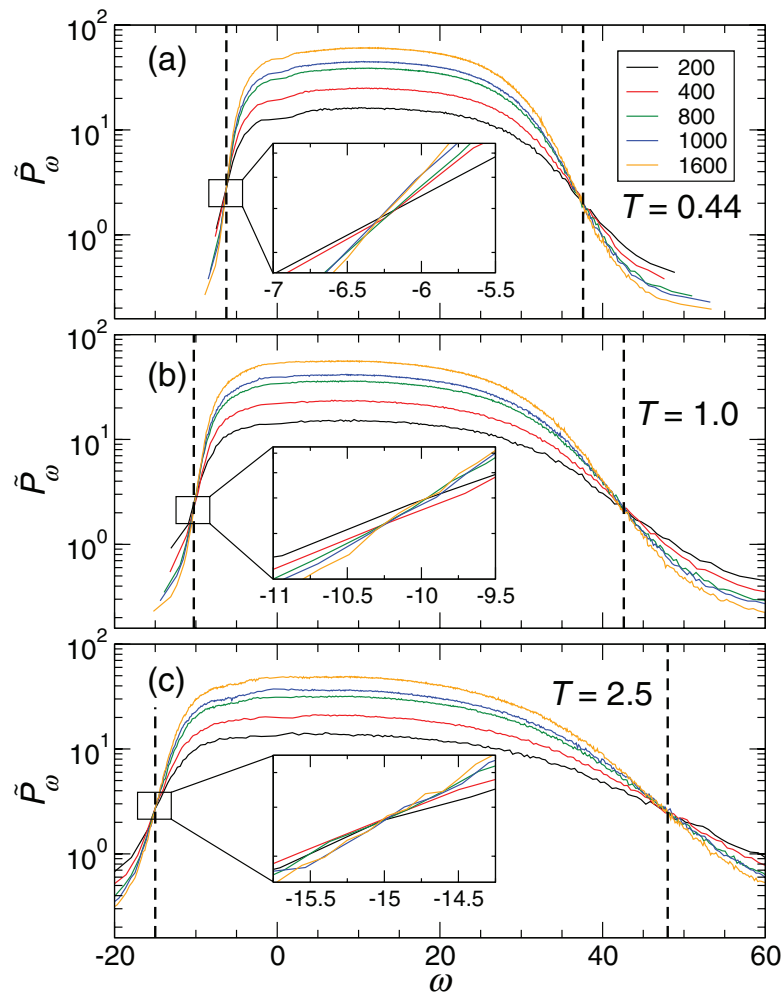

FIG. 3. Modified participation number, $\widetilde{P}_{\omega} \equiv P_{\omega} / L$, for various system sizes at three representative $T$. $\widetilde{P}_{\omega}$ is scale invariant at the crossover between localized and delocalized modes. The dotted lines indicate the boundaries between localized and delocalized modes. The insets show an enlargement of the crossover region for the unstable (imaginary) modes.

a typical configuration in Fig. 4. In Sec. IV, we focus on the relationship of these delocalized modes to dynamics.

The traces of extended or localized behavior of the INMs should also be identifiable in the statistical properties of the eigenfrequencies. ${ }^{51} \mathrm{~A}$ simple spectral measure is the frequency spacing distribution $P(s)$ of the "unfolded" frequency spacings $s_{n}=\left(\omega_{n+1}-\omega_{n}\right) / \Delta$ (where $\Delta=\left\langle\omega_{n+1}-\omega_{n}\right\rangle$ is the mean frequency spacing). In the extended regime, the level statistics should be described quite well by RMT. ${ }^{24,25}$ In particular, the distribution $P(s)$ of the level spacings $s$ should

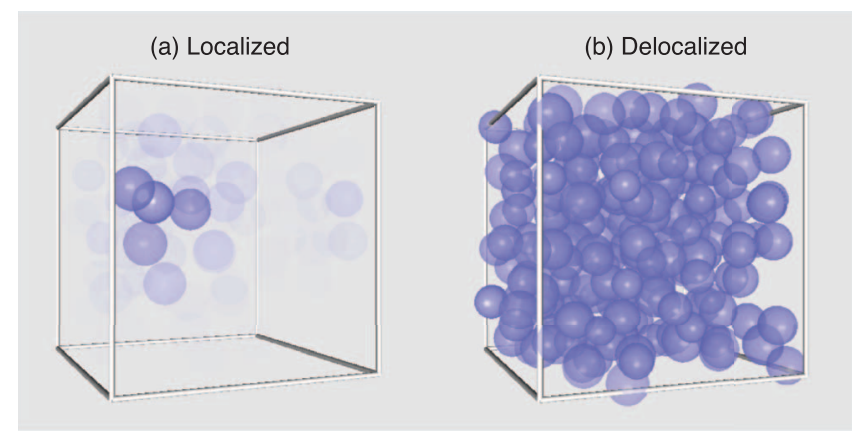

FIG. 4. Visualization of a typical (a) localized and (b) delocalized mode for $N=200$. Particle opacity is determined by $\mathbf{e}_{i} \cdot \mathbf{e}_{i}$, with particles having the larger dot product rendered more opaque. The configuration is identical in both panels, so that the extent of the modes and the participation of specific particles can be compared.
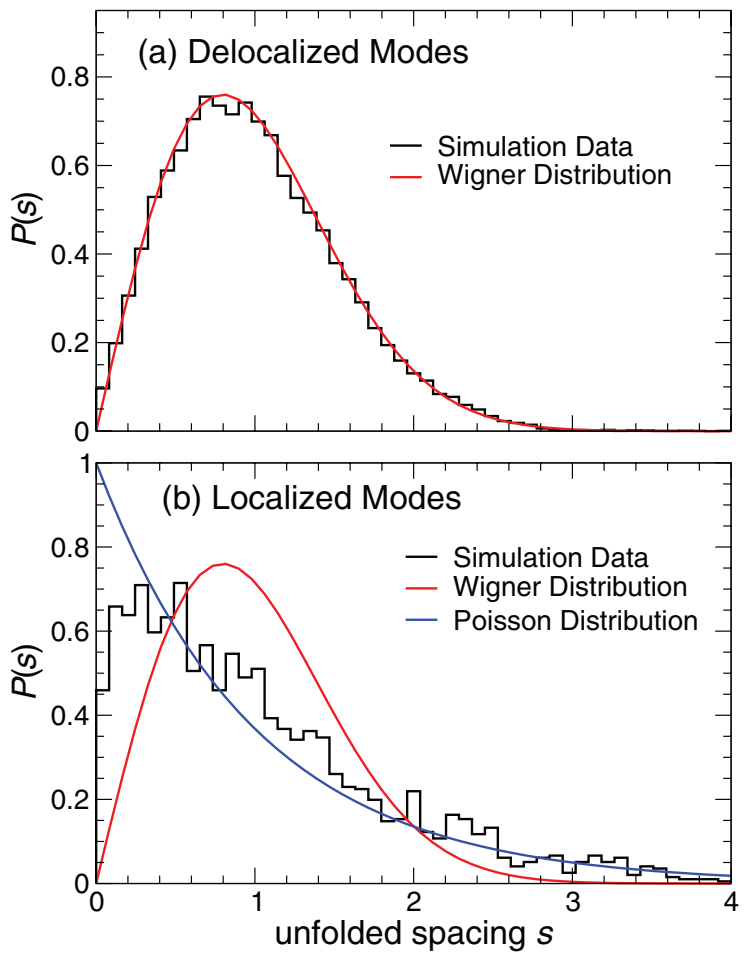

FIG. 5. The distribution $P(s)$ of unfolded frequency spacings $s$ limited to a frequency range where we see either (a) delocalized or (b) localized behavior, demonstrating the expected transition from the Poisson to Wigner behavior. The data shown are for $T=0.44$.

follow the Wigner surmise,

$$
P(s)=\frac{\pi}{2} s \exp \left(-\frac{\pi}{4} s^{2}\right) .
$$

In contrast, in the localized regime, the levels are uncorrelated, leading to a Poisson level spacing distribution.

$$
P(s)=\exp (-s) \text {. }
$$

Previous works ${ }^{22,23}$ found that the mode spacings in the central part of the INM spectrum follow the Wigner distribution and that the spacings near the tails of the spectrum follow Poisson statistics, but neither could precisely locate the MIT crossover point based on the spacing statistics.

Figure 5 confirms the expectations for $P(s)$; specifically, by evaluating $P(s)$ separately on each side of the MIT (determined from the finite scaling behavior of the participation number) there are, indeed, two distinct types of unstable INMs reflected in different $P(s)$ behavior. A detailed analysis of the $P(s)$ behavior was done recently in Refs. 52 and 53, using extremely large systems. Following a standard procedure to distinguish the MIT cross over, ${ }^{51}$ these authors studied the second moment of the nearest neighbor spacings $\left\langle s^{2}\right\rangle$ for a monoatomic Lennard-Jones (LJ) system and showed that approaching the thermodynamic limit $(N \rightarrow \infty)$, there is a transition frequency for which $\left\langle s^{2}\right\rangle$ is scale invariant. However, these authors did not investigate in detail the relation between the fraction of delocalized modes and dynamics. For our system sizes, the fixed point of $\left\langle s^{2}\right\rangle$ is overwhelmed by noise, making this method inappropriate for small systems. We stress that the traditional analysis used by Refs. 52 and 53 required system sizes as large as $\mathcal{O}\left(10^{5}\right)$, which are not 

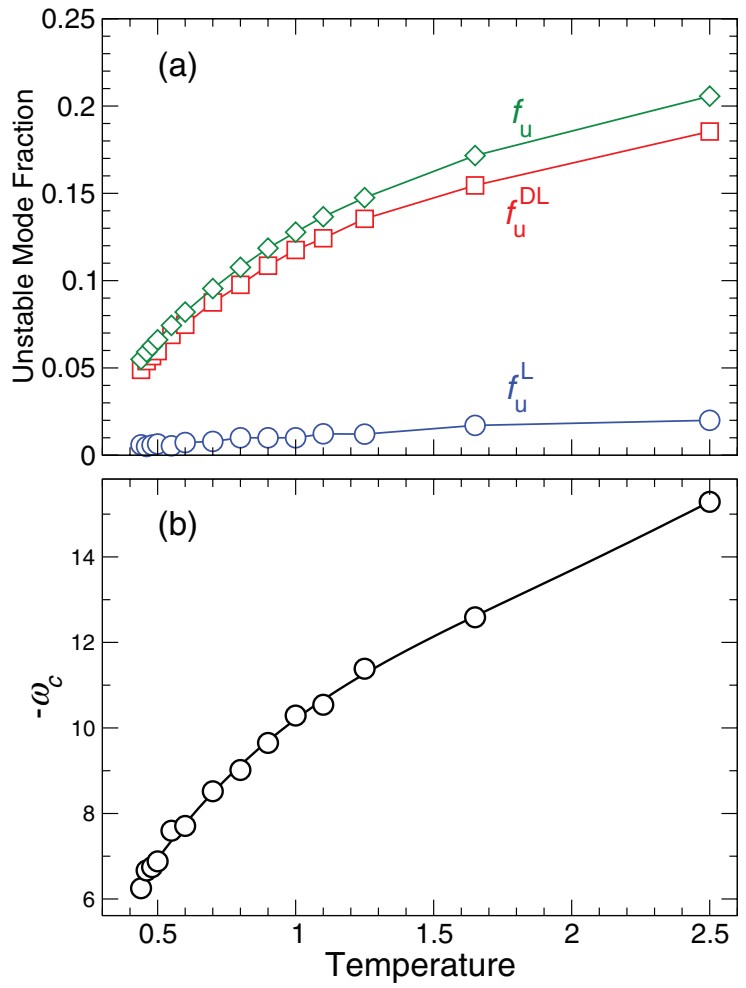

FIG. 6. (a) Unstable mode fractions for all $T$ studied. (b) $T$ dependence of crossover frequency $\omega_{c}$ between localized and delocalized modes, as determined from the participation number. The line is intended only as a guide (Fig. 3).

practical for computational study in deeply supercooled states. In contrast, our PN scaling approach allows us to identify the MIT with relatively small system sizes (of order $\left.\mathcal{O}\left(10^{3}\right)\right)$.

\section{MODE LOCALIZATION AND DYNAMICS}

We first examine the temperature dependence of the fraction of each mode type. Specifically, we first partition the spectrum into the fraction of real, stable modes $f_{\mathrm{s}}$, and the imaginary, unstable modes $f_{\mathrm{u}}$. Obviously, $f_{\mathrm{u}}+f_{\mathrm{s}}=1$. Using the technique to separate localized and delocalized modes discussed in Sec. III, we further split $f_{\mathrm{u}}$ into the delocalized part, $f_{\mathrm{u}}^{\mathrm{DL}}$, and the localized part, $f_{\mathrm{s}}^{\mathrm{L}}$, such that $f_{\mathrm{u}}=f_{\mathrm{u}}^{\mathrm{DL}}+f_{\mathrm{u}}^{\mathrm{L}}$.

Since we are interested in modes relating to diffusion, we show only unstable fractions in Fig. 6(a). On cooling, the overall number of unstable modes, as well as the localized and delocalized subsets, decrease on cooling; this decrease is qualitatively consistent with the decrease of $D$ on cooling. We also show the $T$-dependence of the cutoff frequency $\omega_{C}$ between localized and delocalized modes (Fig. 6(b)), which decreases (in absolute value) on cooling. This decrease roughly mirrors the narrowing of $\langle\rho(\omega)\rangle$ (Fig. 2) on cooling so that the relative fractions $f_{\mathrm{u}}^{\mathrm{DL}} / f_{\mathrm{u}}$ and $f_{\mathrm{u}}^{\mathrm{L}} / f_{\mathrm{u}}$ are nearly invariant with $T$. Note that if $\omega_{c}$ were to vanish at a non-zero $T$, all unstable modes would be localized, possibly coinciding with an ideal definition of $T_{g}$.

We next consider how the delocalized unstable modes compare to earlier efforts to filter the unstable

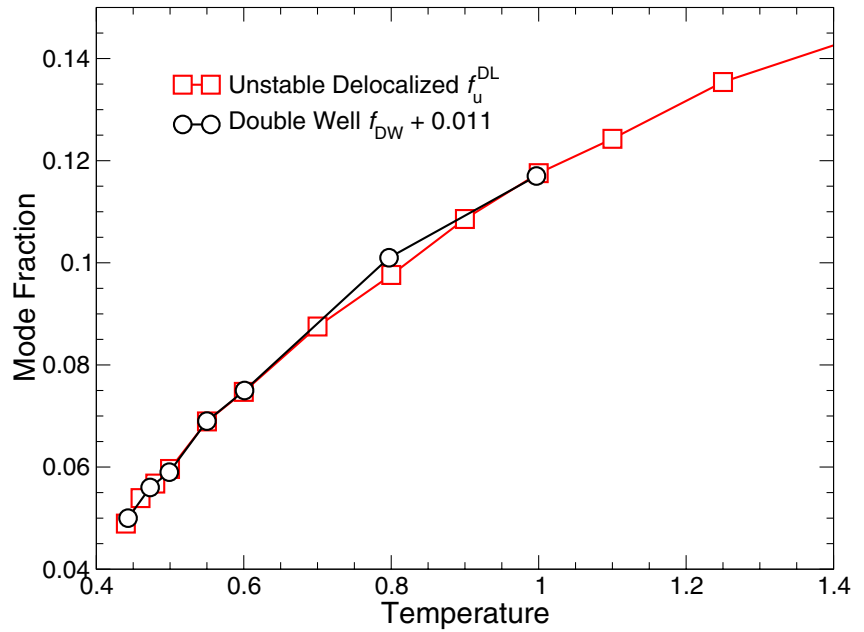

FIG. 7. Comparison of the fraction of unstable delocalized modes $f_{\mathrm{u}}^{\mathrm{DL}}$ with the fraction of double-well modes $f_{\mathrm{DW}}$, showing that they have the same $T$ dependence. Data for $f_{\mathrm{DW}}$ are taken from Ref. 35 and shifted by 0.011 (independent of temperature), such that the data overlap.

modes. ${ }^{12-14,17-19,35}$ One of the most successful alternate approaches to decompose the unstable fraction is to classify the modes based on their potential energy profile, and identify those modes that lead to a different local minimum in the potential energy landscape, which are termed double-well modes $f_{\text {DW }}$. Since diffusion constitutes transitions among such basins of the PES, ${ }^{54,55}$ double-well modes are expected to relate to diffusion. Figure 7(a) compares $f_{\mathrm{u}}^{\text {DL }}$ with the fraction $f_{\mathrm{DW}}$ ( $f_{\mathrm{DW}}$ was calculated in Ref. 35 ). Although $f_{\mathrm{DW}}$ underestimates $f_{\mathrm{u}}^{\mathrm{DL}}$, a small $T$-independent shift (0.011) shows that the two fractions have identical $T$ dependence. Hence, it appears that either classification provides the same information on the $T$ dependence of modes potentially related to diffusion.

Having established an approach that allows us to unambiguously partition localized and delocalized modes, we finally wish to consider to what degree the delocalized modes

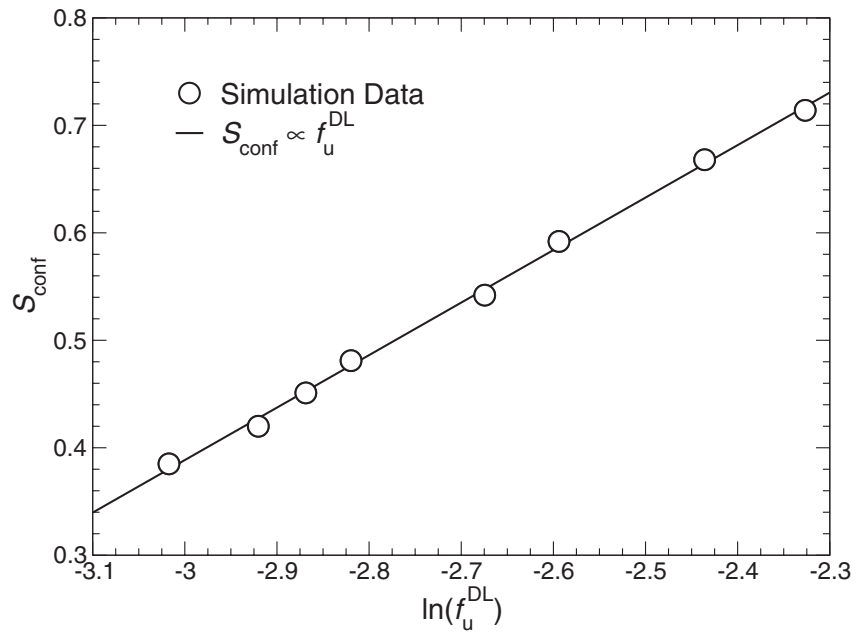

FIG. 8. Demonstration of the proportionality between $S_{\text {conf }}$ and $f_{\mathrm{u}}^{\mathrm{DL}}$. Data for $S_{\text {conf }}$ are taken from Ref. 34. The range of the comparison is limited by the range of $S_{\text {conf }}$ data in Ref. 34. 


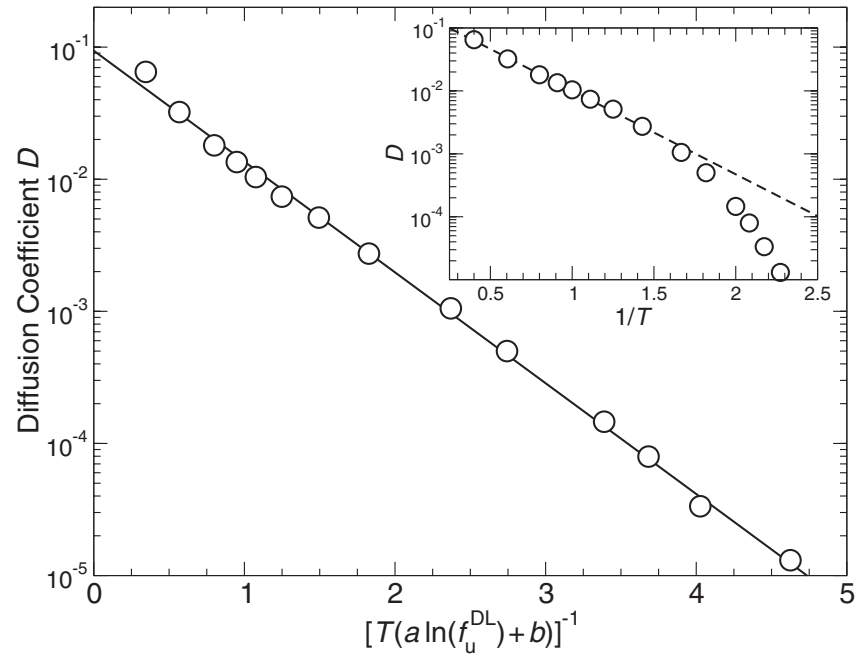

FIG. 9. Diffusion coefficient $D$ as a function of $\left[T\left(a \ln \left(f_{\mathrm{u}}^{\mathrm{DL}}\right)+b\right)\right]^{-1}$. Here, $a=0.5$ and $b=2.0$. The solid line shows the quality of the relation. The inset shows $D$ as a function of $1 / T$ to show the data span the region of both Arrhenius and non-Arrhenius $T$ dependence; the dotted line of the inset is the fit of the high $T$ data with an Arrhenius form.

can account for the $T$ dependence of diffusion. Since $f_{\mathrm{u}}^{\mathrm{DL}}$ and $f_{\text {DW }}$ appear closely related, we consider what is already known about $f_{\text {DW }}$. In a study on supercooled water, Ref. 18 found that $f_{\mathrm{DW}}$ is linearly proportional the number of basins $N_{\mathrm{b}}$ in the potential energy landscape of the system, implying a oneto-one correspondence between basins and transition states that strongly constrains the geometry of the PES. In the PES framework, $N_{b}$ accessed at any given $T$ defines a configurational entropy $S_{\text {conf }}(T)=k_{\mathrm{B}} \ln N_{\mathrm{b}}$. Given the relation between $f_{\mathrm{u}}^{\mathrm{DL}}$ and $f_{\mathrm{DW}}$, the findings of Ref. 18 suggest a linear relation

$$
S_{\mathrm{conf}}=a \ln f_{\mathrm{u}}^{\mathrm{DL}}+b,
$$

where $a$ and $b$ are constants. Keyes ${ }^{56}$ also argued for the applicability of such a relation for the broad class of supercooled liquids using a random energy model.

The configurational entropy for this system has been calculated in Refs. 34 and 36, so that we can directly test Eq. (7); we find such a proportionality holds for the BLJ system (Fig. 8). This provides us with a direct link to the dynamics, since the Adam-Gibbs theory relates $S_{\text {conf }}$ and the diffusion coefficient $D$, namely

$$
D \sim \exp \left[-A /\left(T S_{\text {conf }}\right)\right],
$$

where $A$ is a constant activation free energy. This relation has previously been shown to be valid for the BLJ system ${ }^{36}$ (as well as many other supercooled liquids), so combining with Eq. (7) we expect that

$$
D \sim \exp \left[\frac{A}{T\left(a \ln f_{\mathrm{u}}^{\mathrm{DL}}+b\right)}\right] .
$$

To test this possibility, we evaluate $D$ from the asymptotic behavior of the mean-squared displacement. Figure 9 shows that Eq. (9) holds remarkably well over the entire $T$ range studied, spanning both Arrhenius and non-Arrhenius behavior of $D$ (see the inset of Fig. 9). Figure 9 provides a significant quantitative step toward relating the localization properties
- determined solely from the instantaneous configurationsto dynamics for supercooled liquids. This result might be used to help construct a more refined theoretical description of the dynamics based entirely on system structure and thermodynamics.

Finally, we mention that we have considered several possible direct relations between $D$ the unstable or unstabledelocalized modes. ${ }^{19,42}$ These alternate relations do not appear to describe our data for the binary Lennard-Jones system.

\section{DISCUSSION AND CONCLUSION}

Borrowing ideas developed in the framework of wave (Anderson) localization in random media, we have introduced an approach to unambiguously separate delocalized from localized INM. Focusing on the unstable, delocalized INM, we have shown that the fraction of such modes has a close correspondence to the fraction of doubled-well modes connecting local minima of the PES sampled by a liquid. Motivated by the relation between double-well modes and diffusion, we connected the diffusion coefficient directly with the fraction of unstable, delocalized modes, spanning both Arrhenius and non-Arrhenius regimes, supporting the generality of the relation.

The separation of the unstable INMs to extended and localized was done based on a scaling theory of the participation number, which defines the number of particles participating in a mode. We found that the transition point between extended and localized unstable modes is scale-invariant and can be evaluated accurately with a surprisingly small number of particles. Our results for the transition between extended and localized INMs are consistent with other measures based on statistical analysis of eigenfrequencies of the Hessian.

We should be careful to point out that our relation between $D$ and $f_{\mathrm{u}}^{\mathrm{DL}}$ is phenomenological, and relies on an ansatz that the delocalized modes should be those responsible for diffusion. Thus, we cannot rule out other possible scenarios. For example, since the localized modes are not stationary in time, and since there are $\mathcal{O}(N)$ such modes, it is possible that diffusion could occur through a sequence localized mode excitations. To address these concerns, we intend to build on our approach to develop a more fundamental and testable theoretical relation of dynamics to thermodynamical properties of a liquid via a tomography of the INMs.

\section{ACKNOWLEDGMENTS}

We thank T. Keyes, S. Sastry, F. Sciortino for helpful discussions. V.I.C. thanks F. Vargas Lara and B. Pazmiño for their hospitality during completion of the work. We thank National Science Foundation (NSF) Grant No. CNS-0959856 for support.

${ }^{1}$ P. G. Debenedetti, Metastable Liquids (Princeton University Press, Princeton, NJ, 1996).

${ }^{2}$ W. Götze and L. Sjogren, Rep. Prog. Phys. 55, 241 (1992).

${ }^{3}$ P. G. Debenedetti and F. H. Stillinger, Nature (London) 410, 259 (2001).

${ }^{4}$ T. A. Weber and F. H. Stillinger, Phys. Rev. A 25, 978 (1982).

${ }^{5}$ F. H. Stillinger, Science 267, 1935 (1995). 
${ }^{6}$ S. Sastry, P. Debenedetti, and F. Stillinger, Nature (London) 393, 554 (1998).

${ }^{7}$ A. Rahman, M. J. Mandell, and J. P. McTague, J. Chem. Phys. 64, 1564 (1976).

${ }^{8}$ T. Keyes, J. Phys. Chem. A 101, 2921 (1997).

${ }^{9}$ R. LaViolette and F. H. Stillinger, J. Chem. Phys. 83, 4079 (1985).

${ }^{10}$ R. Cotterill and U. Masden, Phys. Rev. B 33, 262 (1986).

${ }^{11}$ B. Madan and T. Keyes, J. Chem. Phys. 98, 3342 (1993).

${ }^{12}$ S. D. Bembenek and B. B. Laird, Phys. Rev. Lett. 74, 936 (1995).

${ }^{13}$ S. D. Bembenek and B. B. Laird, J. Chem. Phys. 104, 5199 (1995).

${ }^{14} \mathrm{~F}$. Sciortino and P. Tartaglia, Phys. Rev. Lett. 78, 2385 (1997).

${ }^{15}$ W.-X. Li and T. Keyes, J. Chem. Phys. 107, 7275 (1997).

${ }^{16}$ W.-X. Li, T. Keyes, and F. Sciortino, J. Chem. Phys. 108, 252 (1998).

${ }^{17}$ J. D. Gezelter, E. Rabani, and B. J. Berne, J. Chem. Phys. 107, 4618 (1997).

${ }^{18}$ E. La Nave et al., Phys. Rev. Lett. 84, 4605 (2000).

${ }^{19}$ E. La Nave et al., Phys. Rev. E 64, 036102 (2001).

${ }^{20}$ J. Chowdhary and T. Keyes, Phys. Rev. E 65, 026125 (2002).

${ }^{21} 50$ Years of Anderson Localization, edited by E. Abrahams (World Scientific, London, 2010).

${ }^{22}$ S. Sastry, N. Deo, and S. Franz, Phys. Rev. E 64, 016305 (2001).

${ }^{23}$ S. Ciliberti and T. S. Grigera, Phys. Rev. E 70, 061502 (2004).

${ }^{24}$ M. L. Mehta, Random Matrices and the Statistical Theory of Energy Levels (Academic, New York, 1991).

${ }^{25}$ H.-J. Stockmann, Quantum Chaos. An Introduction (Cambridge University Press, Cambridge, 1999).

${ }^{26}$ A. D. Mirlin, Phys. Rep. 326, 259 (2000).

${ }^{27}$ Y. Lahini et al., Phys. Rev. Lett. 100, 013906 (2008).

${ }^{28}$ T. Schwartz, G. Bartal, S. Fishman, and M. Segev, Nature (London) 446, 52 (2007).

${ }^{29}$ J. Billy et al., Nature (London) 453, 891 (2008).
${ }^{30}$ M. Mezard, G. Parisi, and A. Zee, Nucl. Phys. B 559, 689 (1999).

${ }^{31}$ W. Kob and H. C. Andersen, Phys. Rev. E 51, 4626 (1995).

${ }^{32}$ W. Kob and H. C. Andersen, Phys. Rev. E 52, 4134 (1995).

${ }^{33}$ T. A. Weber and F. H. Stillinger, Phys. Rev. B 31, 1954 (1985).

${ }^{34}$ F. Sciortino, W. Kob, and P. Tartaglia, Phys. Rev. Lett. 83, 3214 (1999).

${ }^{35}$ C. Donati, F. Sciortino, and P. Tartaglia, Phys. Rev. Lett. 85, 1464 (2000).

${ }^{36}$ S. Sastry, Nature (London) 409, 164 (2001).

${ }^{37}$ D. Frenkel and B. Smit, Understanding Molecular Simulation From Algorithms to Applications (Academic, San Diego, CA, 1996).

${ }^{38}$ B. Madan, T. Keyes, and G. Seeley, J. Chem. Phys. 92, 7565 (1990).

${ }^{39}$ B. Madan, T. Keyes, and G. Seeley, J. Chem. Phys. 94, 6762 (1991).

${ }^{40}$ G. Seely, T. Keyes, and B. Madan, J. Chem. Phys. 95, 3847 (1991).

${ }^{41}$ W.-X. Li and T. Keyes, J. Chem. Phys. 111, 328 (1999).

${ }^{42}$ W.-X. Li and T. Keyes, J. Chem. Phys. 111, 5503 (1999).

${ }^{43}$ S. Ciliberti et al., Phys. Rev. B 71, 153104 (2005).

${ }^{44}$ G. Zhu, H. Yang, C. Yin, and B. Li, Phys. Rev. E 77, 066113 (2008).

${ }^{45}$ A. D. Mirlin and Y. V. Fyodorov, J. Phys. A 77, 2273 (1991).

${ }^{46}$ S. N. Evangelou and E. N. Economou, Phys. Rev. Lett. 68, 361 (1992).

${ }^{47}$ S. N. Evangelou, J. Stat. Phys. 69, 361 (1992).

${ }^{48}$ P. W. Anderson, Phys. Rev. 109, 1492 (1958).

${ }^{49}$ T. Rieth and M. Schreiber, Z. Phys. B 104, 99 (1997).

${ }^{50}$ R. J. Bell, P. Dean, and D. C. Hibbins-Butler, J. Phys. C 3, 2111 (1970).

${ }^{51}$ B. I. Shklovskii et al., Phys. Rev. B 47, 11487 (1993).

${ }^{52}$ B. J. Huang and T.-M. Wu, Phys. Rev. E 79, 041105 (2009).

${ }^{53}$ B. J. Huang and T.-M. Wu, Phys. Rev. E 82, 051133 (2010).

${ }^{54}$ T. B. Schroder, S. Sastry, J. C. Dyre, and S. C. Glotzer, J. Chem. Phys. 112, 9834 (2000).

${ }^{55}$ N. Giovambattista et al., Phys. Rev. E 65, 041502 (2002).

${ }^{56}$ T. Keyes, Phys. Rev. E 62, 7905 (2000). 